\section{Impact of differences between eyes on binocular measures of vision in patients with cataracts}

${ }^{1}$ Evaluation and Clinical Epidemiology Department. Institut Municipal d'Assistència Sanitària (IMAS), Barcelona, Spain

\section{${ }^{2}$ Ophthalmology}

Department. Institut Municipal d'Assistència Sanitària (IMAS),

Barcelona, Spain

Correspondence: M Comas, Servei d'Avaluació i Epidemiologia Clínica, Institut Municipal d'Assistència Sanitaria (IMAS), Passeig Marítim 25-29, 08003

Barcelona, Spain

Tel: + 34932483291 ;

Fax: + 34932483254 .

E-mail: mcomas@

imas.imim.es

Received: 2 September 2005

Accepted in revised form: 19 January 2006 Published online: 3 March 2006

\begin{abstract}
Purpose To assess the association of stereopsis with differences between eyes (better minus worse eye value) and the binocular value of visual acuity and contrast sensitivity, and to analyse binocular summation or inhibition phenomena owing to differences between eyes.

Methods A cohort of 137 patients with bilateral cataracts (visual acuity of 0.3 LogMAR or worse in both eyes) was followed up through first- and second-eye cataract surgery. The patients were recruited from the ophthalmology departments of two teaching hospitals. Visual acuity, contrast sensitivity (monocular and binocular), and stereopsis were measured preoperatively, after first- and second-eye surgery. Multiple linear regression and local correlation analyses were used.

Results Stereopsis was most strongly influenced by visual acuity in the postoperative period after first-eye surgery (standardized coefficients of $\mathbf{0 . 3 8 2}$ for difference between eyes and 0.356 for binocular visual acuity) and by contrast sensitivity in the postoperative period after second-eye surgery (standardized coefficients of 0.353 for the difference between eyes and -0.312 for binocular contrast sensitivity). After first-eye surgery, the correlation of the differences between eyes with stereopsis was stronger (between 0.4 and 0.5 ) when the differences were greater than $0.4 \log$ MAR units for visual acuity. Slight binocular summation was found for contrast sensitivity. Conclusions When assessing the indication for and outcomes of cataract surgery, analysis of visual function should include measures of both eyes, rather than measures of the
\end{abstract}

M Comas' ${ }^{1}$, X Castells ${ }^{1}$, ER Acosta ${ }^{1}$ and J Tuñí ${ }^{2}$

operative eye only, as differences between eyes (better minus worse eye value) may play an important role in binocular measures such as stereopsis.

Eye (2007) 21, 702-707; doi:10.1038/sj.eye.6702305; published online 3 March 2006

Keywords: cataract extraction; visual acuity; contrast sensitivity; binocular vision

\section{Introduction}

The indication for and benefits of cataract surgery are commonly assessed through monocular measures of vision, mainly visual acuity and occasionally contrast sensitivity. ${ }^{1}$ However, because cataracts are mostly bilateral and cataract surgery is usually performed in one eye at a time, cataract surgery significantly affects binocular vision. In this context, some studies have shown that second-eye cataract surgery restores lack of stereopsis. ${ }^{2,3}$ Moreover, lack of stereopsis has been associated with an increased risk of falls in the elderly ${ }^{4}$ and a lower quality of life. ${ }^{5}$

Studies on binocular vision have mainly focused on the negative impact that differences between eyes, owing to surgery in one eye only, may have on binocular measures of vision, such as stereopsis, binocular visual acuity, and binocular contrast sensitivity. ${ }^{4,6,7}$

Binocular summation and inhibition phenomena have been described for visual acuity and contrast sensitivity. ${ }^{7-9}$ These phenomena are based on the difference between the values of the better eye and the binocular value, that is, with both eyes open. Summation is present when the binocular value is higher than the better eye value, and inhibition when it is lower. 
Although closely related to visual acuity, contrast sensitivity has recently been shown to be an important dimension of vision because of its association with an increased risk of motor vehicle crash, ${ }^{10}$ and because it is strongly affected by binocular summation and inhibition phenomena. ${ }^{8}$ Thus, when cataract surgery causes differences between eyes, impairment in contrast sensitivity may play an important role in visual disability.

The impact of surgery in one eye only on binocular summation phenomena is not clear. Small studies in cataract patients ${ }^{7,8,11}$ found evidence of binocular inhibition in contrast sensitivity and visual acuity. However, other populational studies ${ }^{12,13}$ found a high correlation between binocular and the better-seeing eye measures, but differed in their results on the existence of binocular summation and inhibition.

The purpose of this study was to assess the association of stereopsis with differences between eyes and the binocular value of visual acuity and contrast sensitivity in patients with bilateral cataracts throughout the process from first- to second-eye cataract surgery. Additionally, the presence of binocular summation or inhibition phenomena, and their relationship with differences between eyes, was analysed.

\section{Methods}

\section{Patients}

Patients were recruited from the ophthalmology departments of two teaching hospitals in Barcelona, Spain. Inclusion criteria were assessed by one ophthalmologist in each hospital. Patients were eligible if they were scheduled for first-eye cataract surgery and presented bilateral indication for cataract surgery (visual acuity worse than $0.3 \log$ Mar in both eyes). Exclusion criteria were severe ocular comorbidity (eg, terminal glaucoma, amblyopia, prior strabismus surgery, phoria, or tropia), surgery combined with any other ophthalmologic procedure (eg, glaucoma or keratoplasty), and major complications after first-eye surgery. The study followed the tenets of the Declaration of Helsinki, was approved by the research ethics committees of the two participating centres and patients gave informed consent before being enrolled in the study.

Patients underwent first-eye surgery between 1 and 2 months after enrolment, and second-eye surgery between 2 and 4 months after first-eye surgery. The surgery protocol (ambulatory surgery, phacoemulsification technique with topical anaesthesia, 3.2-mm clear corneal incision, and foldable lens without suture) was identical in both hospitals.

\section{Assessments}

All patients were assessed 1 month before first-eye surgery (baseline), 2 months after first-eye surgery, and 2 months after second-eye surgery. Identical vision tests were used in the two participating hospitals, and were administered by experienced optometrists (one in each hospital).

\section{Vision tests}

LogMar best-corrected visual acuity was measured monocularly and binocularly with an ETDRS chart, calibrated for an 8-f (approximately $2.5 \mathrm{~m}$ ) distance, by the letter-by-letter scoring method. ${ }^{14}$ Log contrast sensitivity was also measured monocularly and binocularly with a Pelli-Robson chart, by the letter-byletter scoring method, ${ }^{15}$ at $1 \mathrm{~m}$. Stereopsis was assessed with the Titmus circles test, which, to widen the range of values, was combined with the Fly and the TNO tests, giving a range from 3000 to $15 \mathrm{~s}$ of arc; an arbitrary value of $4500 \mathrm{~s}$ arc was assigned to the 'non-measurable' category. The measures of stereopsis were also transformed into a log scale. Lower values of visual acuity and stereopsis and higher values of contrast sensitivity indicate good vision. Differences between eyes were calculated as positive values for both visual acuity and contrast sensitivity. Sociodemographic characteristics were obtained by the optometrist at the baseline visit.

\section{Statistical analysis}

The binocular ratio was calculated as the ratio between the binocular value and the better eye value for visual acuity and contrast sensitivity. However, for visual acuity, the decimal scale was used instead of the LogMAR scale to avoid dividing by zero.

The association of stereopsis with visual acuity and contrast sensitivity (binocular and the difference between eyes) was analysed through multiple linear regression, preoperatively, after first- and second-eye surgery. The standardized regression coefficients allow variables to be compared, and were used to determine which variables had the greatest influence on stereopsis. An equivalent analysis was performed using changes in all measures owing to each surgery.

Because the magnitude of association may vary depending on the level of visual impairment, it was checked graphically through local correlation analysis, that is, the association may be more or less intense depending on the value of one of the variables. For example, the association between stereopsis and visual acuity may be more intense at better visual acuities, as 
stereopsis needs a minimum level of visual acuity. Figure 1 shows the local correlation between stereopsis and binocular visual acuity and the difference between eyes after first-eye surgery.

To analyse possible phenomena of binocular summation or inhibition, associations of the better eye measure and the difference between eyes with the binocular measure were assessed by multiple linear regression analysis for visual acuity and contrast sensitivity separately.

\section{Results}

A total of 137 patients were included in the study. The mean age was 71.4 years (standard deviation 9.2) and $62 \%$ were women. Stereopsis, binocular visual acuity,

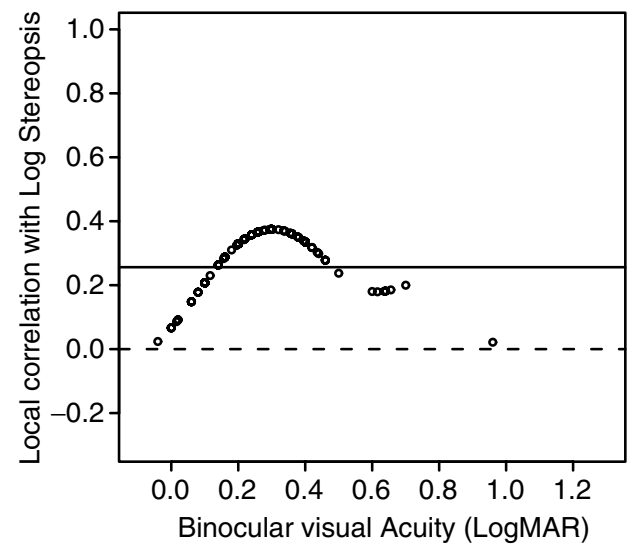

and binocular contrast sensitivity improved after firstand second-eye surgery (Table 1). First-eye surgery was associated with greater improvements in binocular visual acuity and binocular contrast sensitivity, and also with increased differences between eyes. Second-eye surgery showed greater improvements in stereopsis and in reduction of discrepancies between eyes, although stereopsis also improved after first-eye surgery. The binocular ratio showed summation phenomena for contrast sensitivity preoperatively and after first-eye surgery, as its value was greater than 1 (Table 1).

Before all surgery (the patients had bilateral cataracts), binocular contrast sensitivity and the difference between eyes in visual acuity had the greatest influence on stereopsis (Table 2, standardized regression coefficients

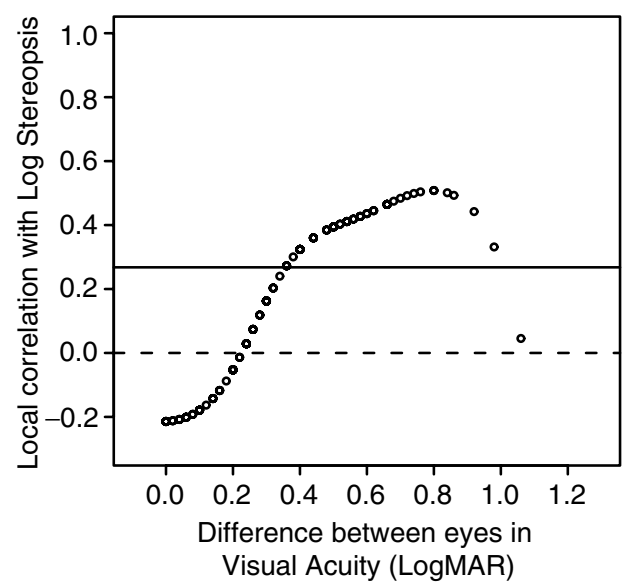

Figure 1 Local correlations of log stereopsis with binocular visual acuity (left) and the difference between eyes in visual acuity (right). Results after first-eye surgery $(n=137)$ are shown. Solid horizontal lines represent overall correlations.

Table 1 Description of the sample throughout the process of cataract removal $(n=137)$

\begin{tabular}{lccc}
\hline & $\begin{array}{c}\text { Preoperative } \\
\text { First-eye surgery }\end{array}$ & $\begin{array}{c}\text { Postoperative } \\
\text { First-eye surgery }\end{array}$ & $\begin{array}{c}\text { Postoperative } \\
\text { Second-eye surgery }\end{array}$ \\
\hline Visual acuity (LogMAR) & & & \\
$\quad$ Binocular visual acuity & $0.54(0.16)$ & $0.25(0.16)$ & $0.12(0.10)$ \\
Better eye & $0.54(0.16)$ & $0.26(0.16)$ & $0.12(0.10)$ \\
Worse eye & $0.72(0.24)$ & $0.61(0.20)$ & $0.23(0.18)$ \\
Difference between eyes & $0.18(0.21)$ & $0.36(0.23)$ & $0.10(0.14)$ \\
Binocular ratio (binocular/better eye) & $1.01(0.12)$ & $1.01(0.12)$ & $1.003(0.03)$ \\
Contrast sensitivity (Log) & & & $1.61(0.12)$ \\
Binocular contrast sensitivity & $1.14(0.29)$ & $1.50(0.23)$ & $1.59(0.13)$ \\
Better eye & $1.10(0.29)$ & $1.46(0.23)$ & $1.49(0.23)$ \\
Worse eye & $0.74(0.46)$ & $0.02(0.39)$ & $0.11(0.16)$ \\
Difference between eyes & $0.36(0.41)$ & $1.03(0.39)$ & $1.01(0.03)$ \\
Binocular ratio (binocular/better eye) & $1.06(0.16)$ & $2.45(0.73)$ & $1.85(0.39)$ \\
Stereopsis (log sec arc) & & 140 & 60 \\
Median stereopsis (sec arc) & $2.88(0.66)$ & 800 & \\
\hline
\end{tabular}

Cell content: mean (standard deviation). 
Table 2 Association of stereopsis with binocular visual acuity and contrast sensitivity and the difference between eyes throughout the process of cataract removal $(n=137)$

\begin{tabular}{|c|c|c|c|c|c|c|c|c|c|}
\hline & \multicolumn{3}{|c|}{ Preoperative first eye } & \multicolumn{3}{|c|}{ Postoperative first eye } & \multicolumn{3}{|c|}{ Postoperative second eye } \\
\hline & Coefficient & Beta $^{\mathrm{a}}$ & $\mathrm{P}$ & Coefficient & Beta $^{\mathrm{a}}$ & $\mathrm{P}$ & Coefficient & Beta $^{\mathrm{a}}$ & $\mathrm{P}$ \\
\hline Constant & 3.391 & & $<0.001$ & 2.314 & & $<0.001$ & 3.320 & & $<0.001$ \\
\hline \multicolumn{10}{|l|}{ Visual acuity } \\
\hline Binocular & 0.277 & 0.068 & 0.408 & 1.612 & 0.356 & 0.001 & 0.361 & 0.093 & 0.179 \\
\hline Difference between eyes & 1.217 & 0.383 & $<0.001$ & 1.199 & 0.382 & 0.001 & 0.360 & 0.122 & 0.233 \\
\hline \multicolumn{10}{|l|}{ Contrast sensitivity } \\
\hline Binocular & -0.888 & -0.395 & $<0.001$ & -0.543 & -0.168 & 0.084 & -1.019 & -0.312 & $<0.001$ \\
\hline Difference between eyes & 0.370 & 0.229 & 0.027 & 0.257 & 0.138 & 0.183 & 0.936 & 0.353 & 0.001 \\
\hline Adjusted $R^{2}$ & $43.6 \%$ & & & $25.7 \%$ & & & $37.9 \%$ & & \\
\hline
\end{tabular}

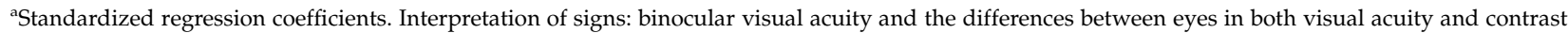
sensitivity were expected to be positive, as higher values represent worse vision, as with log stereopsis. The coefficients of binocular contrast sensitivity were expected to be negative, as a higher value of contrast sensitivity was expected to improve log stereopsis, that is, to reduce its value.

Table 3 Association of change in stereopsis with changes in binocular visual acuity and contrast sensitivity and the difference between eyes throughout the process of cataract removal $(n=137)$

\begin{tabular}{|c|c|c|c|c|c|c|}
\hline & \multicolumn{3}{|c|}{ Change due to first-eye surgery } & \multicolumn{3}{|c|}{ Change due to second-eye surgery } \\
\hline & Coefficient & Beta $a^{\mathrm{a}}$ & $\mathrm{P}$ & Coefficient & Beta ${ }^{\mathrm{a}}$ & $\mathrm{P}$ \\
\hline Constant & -0.186 & & 0.135 & -0.042 & & 0.666 \\
\hline \multicolumn{7}{|l|}{ Change in visual acuity } \\
\hline Binocular & 0.650 & 0.151 & 0.117 & 1.134 & 0.264 & 0.002 \\
\hline Difference between eyes & 0.998 & 0.374 & 0.002 & 0.831 & 0.327 & 0.002 \\
\hline \multicolumn{7}{|l|}{ Change in contrast sensitivity } \\
\hline Binocular & -0.770 & -0.267 & 0.005 & -0.538 & -0.153 & 0.050 \\
\hline Difference between eyes & 0.441 & 0.283 & 0.018 & 0.386 & 0.238 & 0.018 \\
\hline Adjusted $R^{2}$ & $26.8 \%$ & & & $25.0 \%$ & & \\
\hline
\end{tabular}

aStandardized regression coefficients. Interpretation of signs: change in binocular visual acuity and in the differences between eyes in both visual acuity and contrast sensitivity were expected to be positive, as lower values represent improvement, as with log stereopsis. The coefficients of change in binocular contrast sensitivity were expected to be negative, as a higher value of change in binocular contrast sensitivity was expected to cause a lower change in log stereopsis.

of -0.395 and 0.383 , respectively). Visual acuity had the greatest impact on stereopsis in the postoperative period after first-eye surgery (standardized coefficients of 0.382 for the difference between eyes and 0.356 for binocular visual acuity), whereas contrast sensitivity had the greatest influence in the postoperative period after second-eye surgery (standardized coefficients of 0.353 for the difference between eyes and -0.312 for binocular contrast sensitivity).

Improvement in stereopsis after first-eye surgery was associated with a reduction in the difference between eyes in visual acuity (Table 3 , standardized regression coefficient of 0.374 ) and improvement in contrast sensitivity (standardized coefficients of 0.283 for the difference between eyes and -0.267 for binocular contrast sensitivity). Improvement in stereopsis owing to second-eye surgery was most strongly influenced by improvements in visual acuity (standardized coefficient of 0.327 for the difference between eyes and 0.264 for binocular visual acuity), followed by the difference between eyes in contrast sensitivity (0.238) and binocular contrast sensitivity $(-0.153)$.

The local correlation analysis (Figure 1) showed that after first-eye surgery the correlation of the differences between eyes in visual acuity with stereopsis was stronger (correlation coefficient between 0.4 and 0.5) when the differences were greater (especially when more than $0.4 \log$ MAR units). In contrast, no clear tendency was observed for the local correlation between stereopsis and binocular visual acuity, although higher correlations 
Table 4 Association of binocular visual acuity with better eye visual acuity and the difference between eyes throughout the process of cataract removal $(n=137)$

\begin{tabular}{|c|c|c|c|c|c|c|}
\hline & \multicolumn{2}{|c|}{ Preoperative first eye } & \multicolumn{2}{|c|}{ Postoperative first eye } & \multicolumn{2}{|c|}{ Postoperative second eye } \\
\hline & Coefficient & $\mathrm{P}$ & Coefficient & $\mathrm{P}$ & Coefficient & $\mathrm{P}$ \\
\hline Constant & 0.007 & 0.687 & -0.001 & 0.958 & 0.002 & 0.297 \\
\hline Better eye & 0.984 & $<0.001$ & 0.964 & $<0.001$ & 0.974 & $<0.001$ \\
\hline Difference between eyes & 0.003 & 0.901 & 0.019 & 0.315 & 0.007 & 0.278 \\
\hline Adjusted $R^{2}$ & $88.7 \%$ & & $92.3 \%$ & & $99.0 \%$ & \\
\hline
\end{tabular}

Table 5 Association of binocular contrast sensitivity with better eye contrast sensitivity and the difference between eyes throughout the process of cataract removal $(n=137)$.

\begin{tabular}{|c|c|c|c|c|c|c|}
\hline & \multicolumn{2}{|c|}{ Preoperative first eye } & \multicolumn{2}{|c|}{ Postoperative first eye } & \multicolumn{2}{|c|}{ Postoperative second eye } \\
\hline & Coefficient & $\mathrm{P}$ & Coefficient & $\mathrm{P}$ & Coefficient & $\mathrm{P}$ \\
\hline Constant & 0.100 & 0.003 & 0.140 & $<0.001$ & 0.262 & $<0.001$ \\
\hline Better eye & 0.961 & $<0.001$ & 0.942 & $<0.001$ & 0.848 & $<0.001$ \\
\hline Difference between eyes & -0.039 & 0.063 & -0.039 & 0.014 & -0.018 & 0.374 \\
\hline Adjusted $R^{2}$ & $89.0 \%$ & & $90.7 \%$ & & $90.4 \%$ & \\
\hline
\end{tabular}

were observed for intermediate values of binocular visual acuity.

For visual acuity (Table 4) the better eye value had a high correlation with the binocular measure (the only significant coefficient, with a value near one, and the models explained around $90 \%$ of the variance). For contrast sensitivity (Table 5) similar results were obtained, although some influence of the difference between eyes was suggested, especially in the postoperative period after first-eye surgery.

\section{Discussion}

The present study shows that stereopsis is influenced by the difference between eyes, as well as by binocular measures of visual acuity and contrast sensitivity. This association varied at different times during the process of cataract removal, as visual acuity had a greater impact on stereopsis after first-eye surgery, and contrast sensitivity had a stronger impact after second-eye surgery. Slight binocular summation phenomena were observed for contrast sensitivity, whereas none were observed for visual acuity.

Patients with bilateral cataracts who have surgery in both eyes usually undergo cataract removal in one eye at a time. Both visual acuity and contrast sensitivity substantially improve after first-eye surgery and, to a lesser extent, after second-eye surgery. ${ }^{2}$ Thus, first-eye surgery results in good binocular visual acuity and contrast sensitivity, which are strongly influenced by the better eye value. However, this may result in a considerable difference between eyes. The cohort analysed in the present study allowed the effects of differences between eyes on binocular vision to be assessed throughout the process of cataract removal.

An imbalance between eyes may cause impairment in stereopsis. ${ }^{6,16}$ In our study, stereopsis improved after both first- and second-eye surgery, even though no benefit in stereopsis can be expected when surgery leads to a marked difference between eyes. This improvement may be explained by the relationship between stereopsis and binocular visual acuity, mainly present after first-eye surgery, and by marked preoperative impairment, as stereopsis requires a minimum level of vision. ${ }^{17}$ Moreover, the difference between eyes in visual acuity had a marked negative effect on stereopsis when it was higher than $0.4 \log$ MAR units.

A small study ${ }^{18}$ showed that binocular inhibition was present after first-eye surgery, and that restoring good vision in the fellow eye, thus reducing the difference between eyes, led to normal binocular summation. In our study, no important binocular summation or inhibition phenomena were observed for visual acuity, but a slight summation effect for contrast sensitivity was detected preoperatively and after first-eye surgery. This was consistent with the findings of Pardhan et $\mathrm{al}^{7}$ in unilateral cataract patients: binocular contrast sensitivity is influenced by both eyes, whereas binocular visual acuity is influenced only by the better eye measure. 
Our study supports the idea that the decision to operate on the second eye should be based on the outcome of the first surgery. Restoring good vision in one eye alone benefits binocular visual acuity and contrast sensitivity, but when this causes a marked difference between eyes, the impairment in stereopsis acquires greater importance. Two randomized clinical trials showed that the most important outcome of surgery in both eyes, compared with surgery in one eye only, was stereopsis, followed by patient-reported visual disability. ${ }^{2,3}$

A possible limitation of our study is the similarity between eyes related to preoperative visual acuity. Because patients with a visual acuity of $0.3 \log$ MAR or worse in both eyes were selected, the range of preoperative visual acuities might be narrower than that of patients undergoing surgery in both eyes.

This is the first study to assess the associations between stereopsis and visual acuity and contrast sensitivity throughout the process of cataract removal in bilateral cataract patients. Previous studies have mainly focused on patients with unilateral cataracts, with healthy or pseudophakic fellow eyes. Moreover, the measure of stereopsis was more refined than that used in previous studies: several tests were combined to obtain a wider range of values, more approximate to the real value, and the 'non-measurable' category was assigned a more extreme value than those used in other studies. Moreover, this value was analysed through its logarithm, which has more accurate mathematical properties.

In conclusion, when assessing the indication for and outcomes of cataract surgery, analysis of visual function should include measures of both eyes rather than the measure of the operative eye only. Specifically, for visual acuity and contrast sensitivity, monocular measures of the better eye were strongly associated with binocular measures. However, in the case of stereopsis, the difference between eyes and the binocular measure, in both visual acuity and contrast sensitivity, play an important role, which may vary depending on the stage of the process of cataract removal.

\section{Acknowledgements}

This work was supported by grants from the Catalan Agency for Health Technology Assessment and Research (CAHTAR) (10/31/98 and 089/07/2000) and the Fondo de Investigación Sanitaria (FIS) (99/0686, PI020365, and the Networks of Excellence IRYSS G03/202 and RCESP C03/09).

\section{References}

1 Frost NA, Sparrow JM. Use of vision tests in clinical decision making about cataract surgery: results of a national survey. Br J Ophthalmol 2000; 84: 432-434.

2 Castells X, Comas M, Alonso J, Espallargues M, Martínez V, Garcia-Arumí J et al. In a randomised controlled trial, cataract surgery in both eyes increased benefits compared to surgery in one eye only. J Clin Epidemiol 2006; 59: 201-207.

3 Laidlaw DAH, Harrad RA, Hopper CD, Whitaker A, Donovan JL, Brookes ST et al. Randomised trial of effectiveness of second eye cataract surgery. Lancet 1998; 352: 925-929.

4 Nevitt MC, Cummings SR, Kidd S, Black D. Risk factors for recurrent nonsyncopal falls. A productivity study. JAMA 1989; 261: 2663-2668.

5 Kuang TM, Hsu WM, Chou CK, Tsai SY, Chou P. Impact of stereopsis on quality of life. Eye 2005; 19: 540-545.

6 Donzis PB, Rappazzo JA, Borde RM, Gordon M. Effect of binocular variations of Snellen's visual acuity on titmus stereoacuity. Arch Ophthalmol 1983; 101: 930-932.

7 Pardhan S, Elliott DB. Clinical measurements of binocular summation and inhibition in patients with cataract. Clin Vis Sci 1991; 6: 355-359.

8 Taylor RH, Misson GP, Moseley MJ. Visual acuity and contrast sensitivity in cataract: summation and inhibition of visual performance. Eye 1991; 5: 704-707.

9 McElvanney A, Moseley MJ, Jones HS. Binocular inhibition of visual performance in patients with cataract. The influence of test reliability. Acta Ophthalmol Copenh 1994 72: 606-611.

10 Owsley C, McGwin G, Sloane M, Wells J, Stalvey BT, Gauthreaux S. Impact of cataract surgery on motor vehicle crash involvement by older adults. JAMA 2002; 288: 841-849.

11 Pardhan S, Gilchrist J. The importance of measuring binocular contrast sensitivity in unilateral cataract. Eye 1991; 5: 31-35.

12 Rubin GS, Muñoz B, Bandeen-Roche K, West SK, for the SEE Project Team. Monocular versus binocular visual acuity as measures of vision impairment and predictors of visual disability. Invest Ophthalmol Vis Sci 2000; 41: 3327-3334.

13 Azen SP, Varma R, Preston-Martin S, Ying-Lai M, Globe D, Hahn S, for the Los Angeles Latino Eye Study Group. Binocular visual acuity summation and inhibition in an ocular epidemiological study: the Los Angeles Latino Eye Study. Invest Ophthalmol Vis Sci 2002; 43: 1742-1748.

14 Vanden Bosch ME, Wall M. Visual acuity scored by the letter-by-letter or probit methods has lower retest variability than the line assignment method. Eye 1997; 11: 411-417.

15 Elliott DB, Bullimore MA, Bailey IL. Improving the reliability of the Pelli-Robson contrast sensitivity test. Clin Vis Sci 1991; 2: 471-475.

16 Legge GE, Gu Y. Stereopsis and contrast. Vis Res 1989; 29: 989-1004.

17 Kwapiszeski BR, Gallagher CC, Holmes JM. Improved stereoacuity: an indicator for unilateral cataract surgery. J Cataract Refract Surg 1996; 22: 441-445.

18 Laidlaw A, Harrad R. Can second eye cataract extraction be justified? Eye 1993; 7: 680-686. 